Supplement of Geosci. Model Dev., 13, 5663-5685, 2020

https://doi.org/10.5194/gmd-13-5663-2020-supplement

(C) Author(s) 2020. This work is distributed under

the Creative Commons Attribution 4.0 License.

(c) (1)

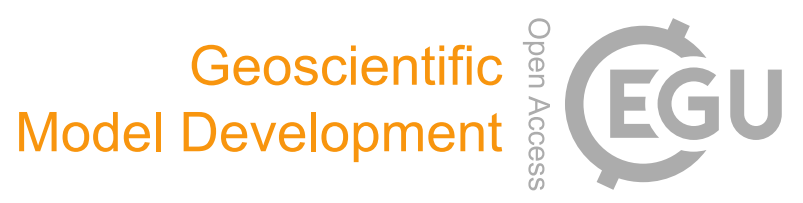

Supplement of

\title{
Sensitivity of spatial aerosol particle distributions to the boundary conditions in the PALM model system 6.0
}

Mona Kurppa et al.

Correspondence to: Mona Kurppa (mona.kurppa@helsinki.fi) and Leena Järvi (leena.jarvi@helsinki.fi)

The copyright of individual parts of the supplement might differ from the CC BY 4.0 License. 


\section{Contents}

S2 Measurements 2

S3 Simulations $\quad 3$

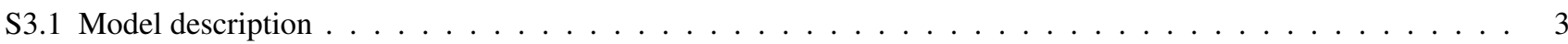

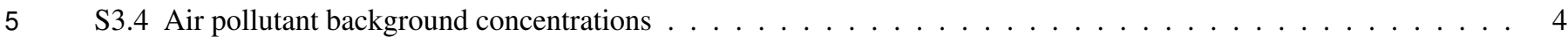

S3.4.1 9 Jun $2017 \ldots \ldots \ldots \ldots \ldots \ldots \ldots \ldots \ldots \ldots \ldots$

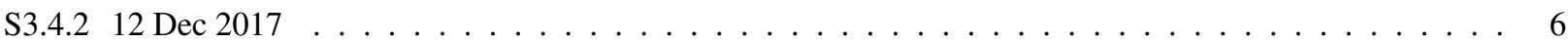

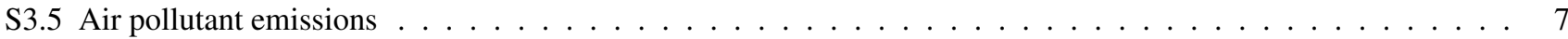

S4 Comparison of the modelled and observed boundary conditions $\quad 7$

10 S5 Evaluation of the air quality modelling results $\quad 12$

S5.2 Horizontal distribution of total aerosol particle number concentration . . . . . . . . . . . . . . . . . . 12

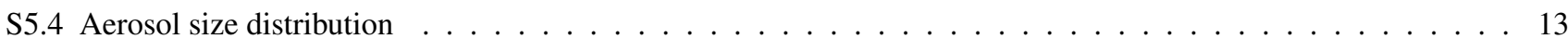

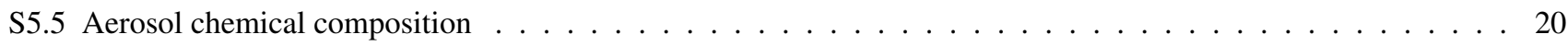

$\begin{array}{lr}\text { S6 Sensitivity analysis } & 22\end{array}$ 


\section{S2 Measurements}

Table S1. Instrumentation. Abbreviations: PSD = aerosol particle size distribution, $\mathrm{BC}=$ black carbon, $\mathrm{PM}_{2.5}=$ particulate matter with aerodynamic diameter $<2.5 \mu \mathrm{m}, \mathrm{LDSA}=$ lung-deposited surface area.

\begin{tabular}{lll}
\hline Site & Variable & Instrument \\
\hline Supersite & PSD (size range 6-800 nm) & $\begin{array}{l}\text { Differential mobility particle sizer (DMPS: A20 CPC, Airmodus } \\
\text { \& Vienna-type Differential Mobility Analyzer (DMA)) }\end{array}$ \\
& Aerosol chemical composition & Aerosol Chemical Speciation Monitor \\
& $\left(\mathrm{SO}_{4}^{-}, \mathrm{OC}, \mathrm{NO}_{3}^{-}, \mathrm{NH}_{4}^{+}, 40-600 \mathrm{~nm}\right)$ & (ACSM, Aerodyne Research) \\
& $\mathrm{BC}(<1 \mu \mathrm{m})$ & $\begin{array}{l}\text { Multi-angle Absorption Photometer (MAAP 5012, } \\
\text { Thermo Scientific Inc.) }\end{array}$ \\
& & Tapered element oscillating microbalance (TEOM 1405, \\
& $\mathrm{PM}_{2.5}$ & Thermo Scientific Inc.) \\
& & Differential mobility particle sizer (DMPS: A20 CPC, Airmodus \\
SMEAR III & PSD $(6-800 \mathrm{~nm})$ & \& Vienna-type Differential Mobility Analyzer (DMA)) \\
& & AQ Urban (Pegasor Oy) \\
& LDSA & 3D ultrasonic anemometer (Metek USA-1, Metek GmbH) \\
& Wind speed and direction & 3D ultrasonic anemometer (Metek USA-1, Metek GmbH) \\
& Air temperature & AQ Urban (Pegasor Oy) \\
\hline
\end{tabular}




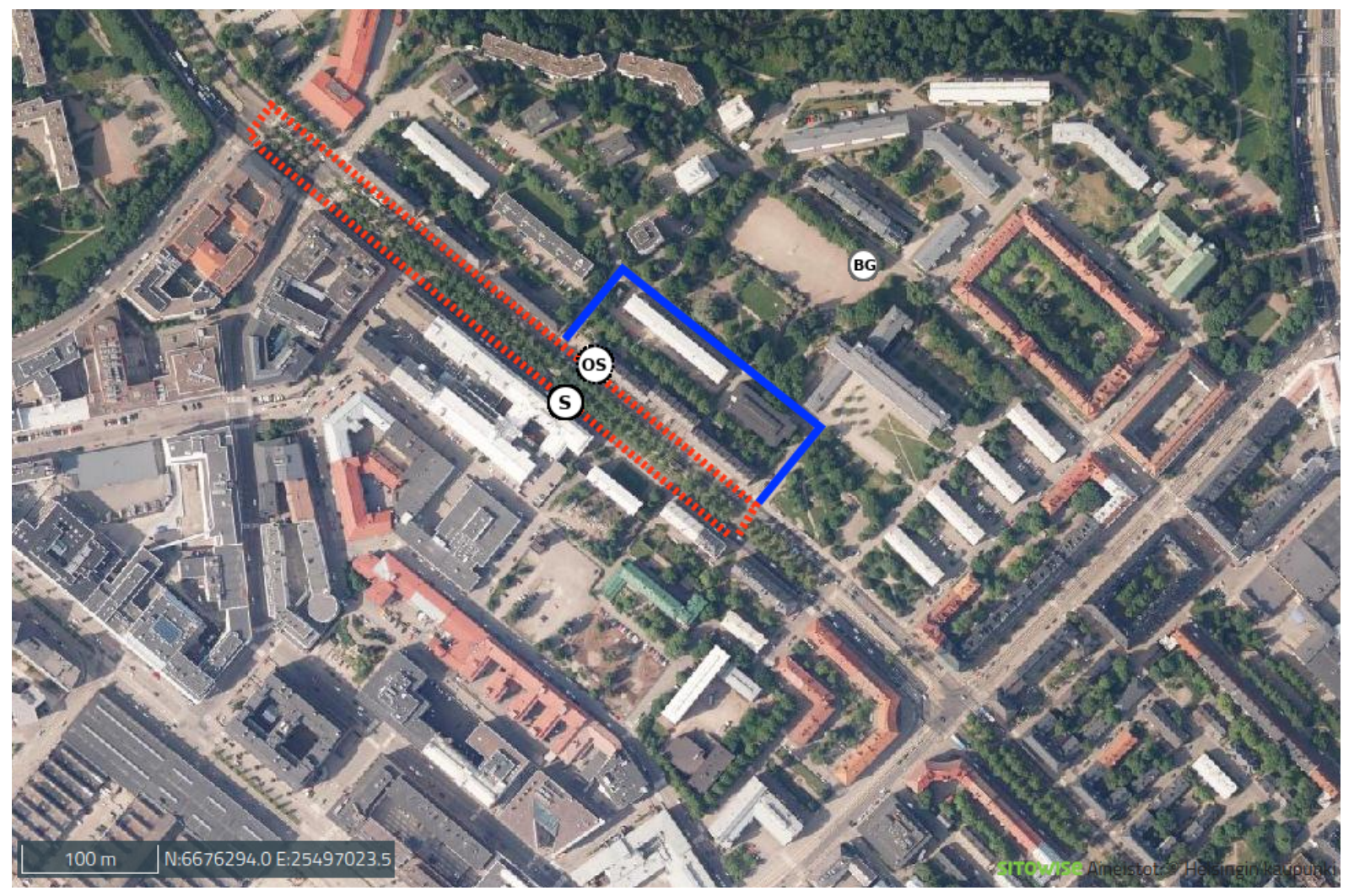

Figure S1. The Sniffer route: main street with a red dotted line and the side street with a blue solid line. Stationary measurement points: $\mathrm{S}=$ supersite, $\mathrm{OS}=$ opposite the supersite, $\mathrm{BG}=$ background. Map from https://kartta.hel.fi.

\section{S3 Simulations}

\section{S3.1 Model description}

The chemical mechanism salsa+simple found in the palm repository (https://palm.muk.uni-hannover.de/trac/browser/ palm/trunk/UTIL/chemistry/gasphase_preproc/mechanisms/def_salsa\%2Bsimple?rev=4416).

$5 \quad \mathrm{NO}_{2}+h v=\mathrm{NO}+\mathrm{O}_{3}$

$\mathrm{O}_{3}+\mathrm{H}_{2} \mathrm{O}=2 \mathrm{OH}$

$\mathrm{NO}+\mathrm{O}_{3}=\mathrm{NO}_{2}$

10

$$
\mathrm{RH}+\mathrm{OH}=\mathrm{RO}_{2}+\mathrm{H}_{2} \mathrm{O}
$$


$\mathrm{HO}_{2}+\mathrm{NO}=\mathrm{NO}_{2}+\mathrm{OH}$

5

$\mathrm{NO}_{2}+\mathrm{OH}=\mathrm{HNO}_{3}$

\section{S3.4 Air pollutant background concentrations}

The trace gas emissions along the ADCHEM trajectories are taken from the Copernicus Atmosphere Monitoring Service global and regional emissions (Granier et al., 2019). For the anthropogenic emissions of carbon monoxide (CO), non-methane volatile organic compounds (NMVOC), nitrous oxides $\left(\mathrm{NO}_{\mathrm{x}}\right.$ ), sulphur dioxide $\left(\mathrm{SO}_{2}\right)$ and $\mathrm{NH}_{3}$ we use the CAMS-REG-v3.1 emission inventory for year 2015, which groups the air pollution emissions into 15 emission-type sectors. The spatial resolution is $0.05^{\circ} \times 0.1^{\circ}$. Over Finland the anthropogenic trace gas emissions are taken from an emission inventory provided by the Finnish Environment Institute (SYKE), which has been derived with the Finnish Regional Emission Scenario model FRES (Karvosenoja, 2008) and has a spatial resolution of $250 \mathrm{~m} \times 250 \mathrm{~m}$. From Finnish power plants and industry, emissions were provided as point sources. The anthropogenic primary particle emissions, apart those from road traffic, are estimated using the size-resolved primary particle emission inventory from Paasonen et al. (2016) with a spatial resolution of $0.5^{\circ} \times 0.5^{\circ}$. For the road traffic, primary particle emissions are estimated using the $\mathrm{NO}_{\mathrm{x}}$ emissions from the road traffic and the temperature dependent road traffic exhaust emission factors derived from measurements in Helsinki (Ripamonti et al., 2013). In order to scale the road traffic exhaust particle emissions based on the $\mathrm{NO}_{\mathrm{x}}$ emission factors an approximate $\mathrm{NO}_{\mathrm{x}}$ emission factor of $1.0 \times 10^{-3} \mathrm{~g}$ vehicle ${ }^{-1} \mathrm{~m}^{-1}$ was applied. This factor is estimated based on the $\mathrm{NO}_{\mathrm{x}}$ vehicle emission factors reported at an urban street in Copenhagen $\left(0.93 \times 10^{-3} \mathrm{~g}\right.$ vehicle $\left.\mathrm{e}^{-1} \mathrm{~m}^{-1}\right)$ and at a highway in Denmark $\left(1.4 \times 10^{-3} \mathrm{~g}_{\text {vehicle }}^{-1} \mathrm{~m}^{-1}\right)($ Wang et al., 2010). The characteristic size-resolved road traffic primary particle emission distribution is taken from Kristensson et al. (2004). Primary particle emissions from ships traffic is parameterized based on the $\mathrm{SO}_{2}$ emissions (Roldin et al., 2019). All anthropogenic emissions are corrected using monthly, weekly and hourly time profiles originally developed for the LOTOSEUROS model (Schaap et al., 2008).

The size resolved marine sea-spray primary particle emissions are calculated using the parameterization from Mårtensson et al. (2003). The dimethyl sulphide emissions are considered using the daily CAMS-OCE global oceanic emissions with a spatial resolution of $0.5^{\circ} \times 0.5^{\circ}$ (Lana et al., 2011). Global soil $\mathrm{NO}_{\mathrm{x}}$ emissions are taken from $0.5^{\circ} \times 0.5^{\circ}$ resolution, monthly emission files for year 2015 (Simpson et al., 2014). The biogenic VOC emissions are modelled using a one-dimensional version of MEGAN 2.04 (Model of Emissions of Gases and Aerosols from Nature Guenther et al., 2006), which has been implemented into ADCHEM (Roldin et al., 2019). 
S3.4.1 9 Jun 2017

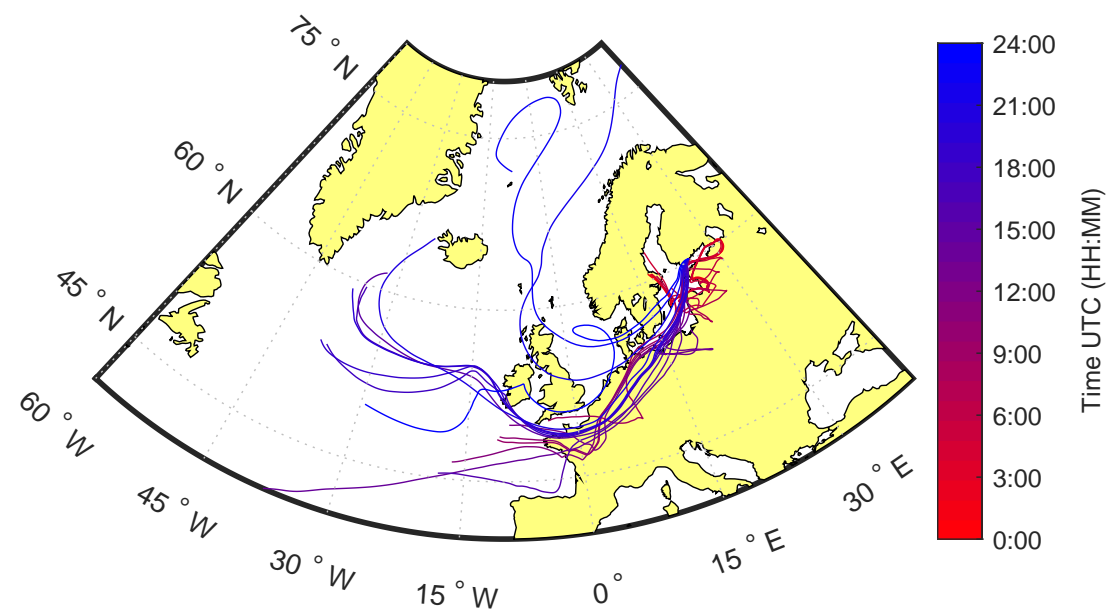

Figure S2. HYSPLIT seven-days-long back trajectories arriving to the supersite on 9 Jun 2017 from 00:00 UTC to 24:00 UTC 
S3.4.2 12 Dec 2017

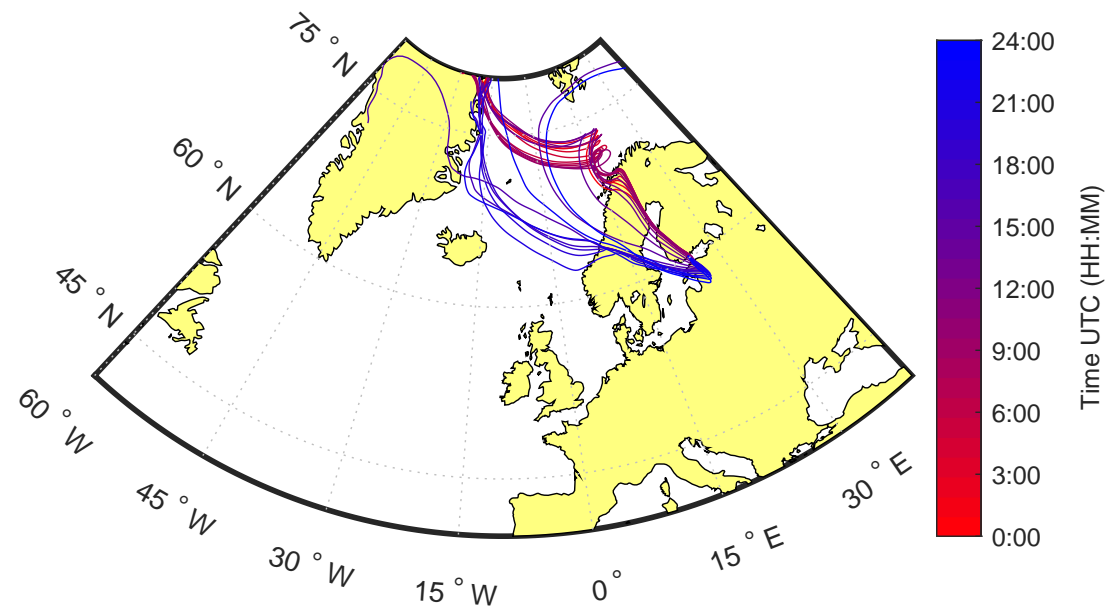

Figure S3. HYSPLIT seven-days-long back trajectories arriving to the supersite on 12 Dec 2017 from 00:00 UTC to 24:00 UTC 


\section{S3.5 Air pollutant emissions}

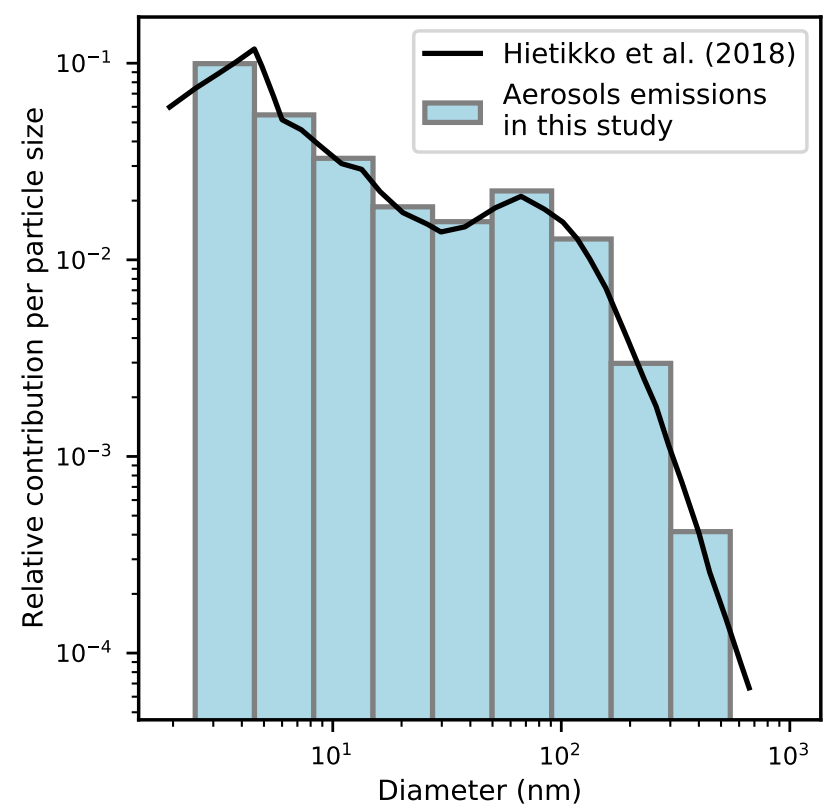

Figure S4. Relative contribution to the aerosol size distribution per particle size for Hietikko et al. (2018) and the traffic-combustion-related aerosol emission applied in this study.

\section{S4 Comparison of the modelled and observed boundary conditions}

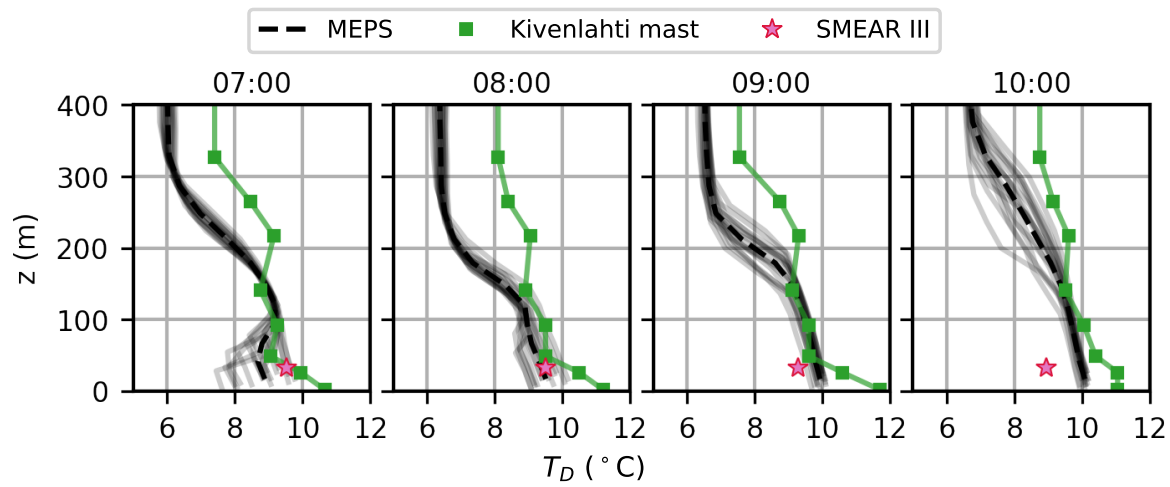

Figure S5. Dew-point temperature $T_{D}\left({ }^{\circ} \mathrm{C}\right)$ on 9 Jun at local time $(\mathrm{UTC}+3)$. The modelled profiles at each MEPS grid point are shown by grey solid lines and their mean by a black dashed line. The observation from the Kivenlahti mast are shown by green solid squares and the interpolated profiles used as boundary conditions by a green solid line. Stars show the SMEAR III observations. 


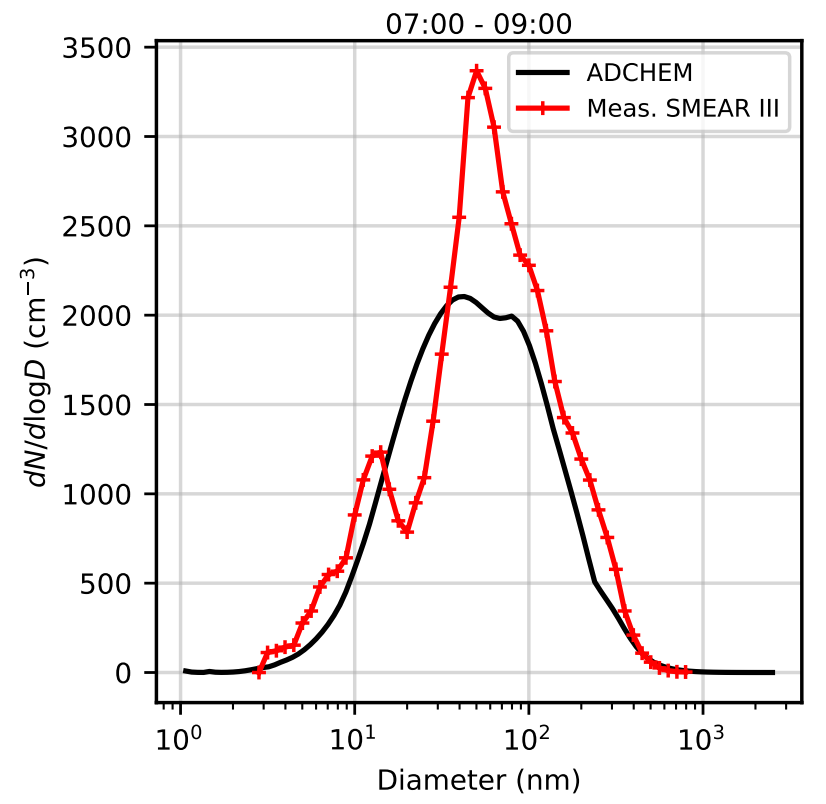

Figure S6. Mean modelled (black solid line) and measured aerosol size distribution at the SMEAR station (red line with crosses) on 9 Jun 2017 between 07:00-09:00 local time. Modelled values are from ADCHEM for a trajectory arriving in Helsinki (60 $\left.12^{\prime} \mathrm{N}, 24^{\circ} 57^{\prime} \mathrm{E}\right)$. 

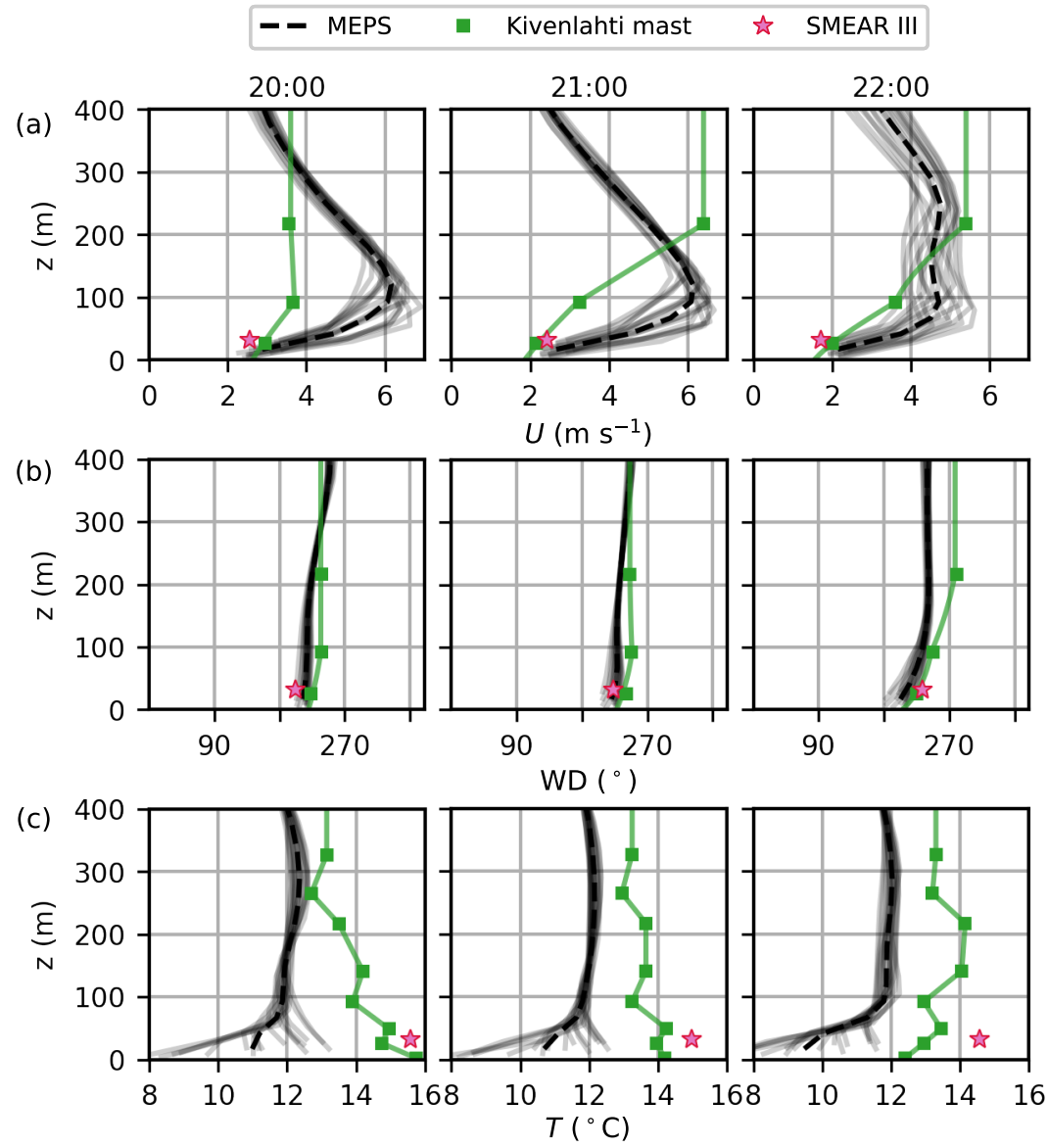

Figure S7. Horizontal a) wind speed $U\left(\mathrm{~m} \mathrm{~s}^{-1}\right)$, b) wind direction WD $\left({ }^{\circ}\right)$ and c) air temperature $T\left({ }^{\circ} \mathrm{C}\right)$ on 9 Jun at local time (UTC+3). See Fig. S5 caption for details.

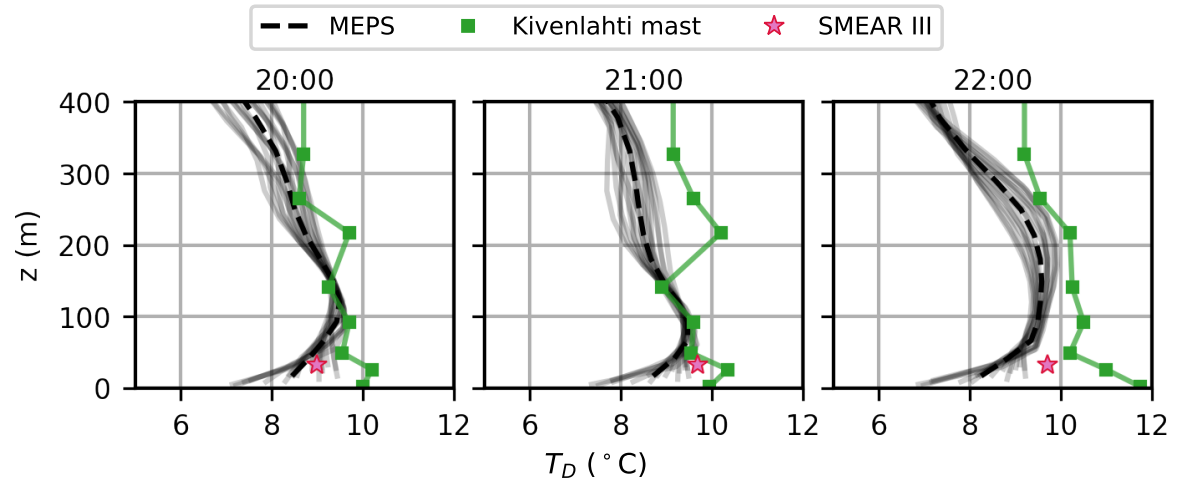

Figure S8. Dew-point temperature $T_{D}\left({ }^{\circ} \mathrm{C}\right)$ on 9 Jun at local time (UTC+3). See Fig. S5 for details. 


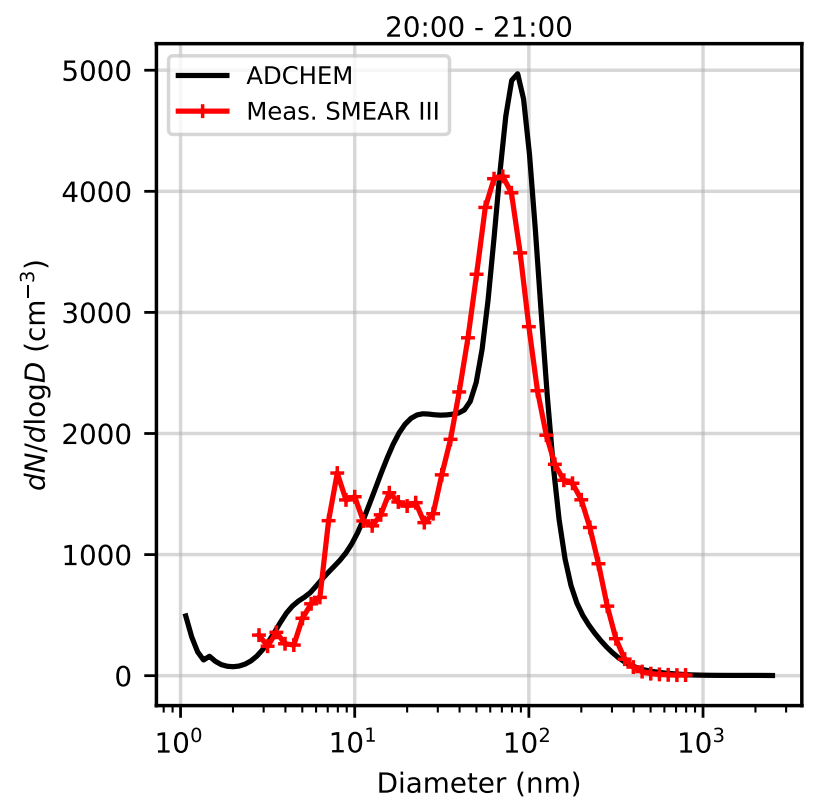

Figure S9. Mean modelled (black solid line) and measured aerosol size distribution at the SMEAR station (red line with crosses) on 9 Jun 2017 between 20:00-21:00 local time. Modelled values are from ADCHEM for a trajectory arriving in Helsinki (60 $\left.12^{\prime} \mathrm{N}, 24^{\circ} 57^{\prime} \mathrm{E}\right)$. 

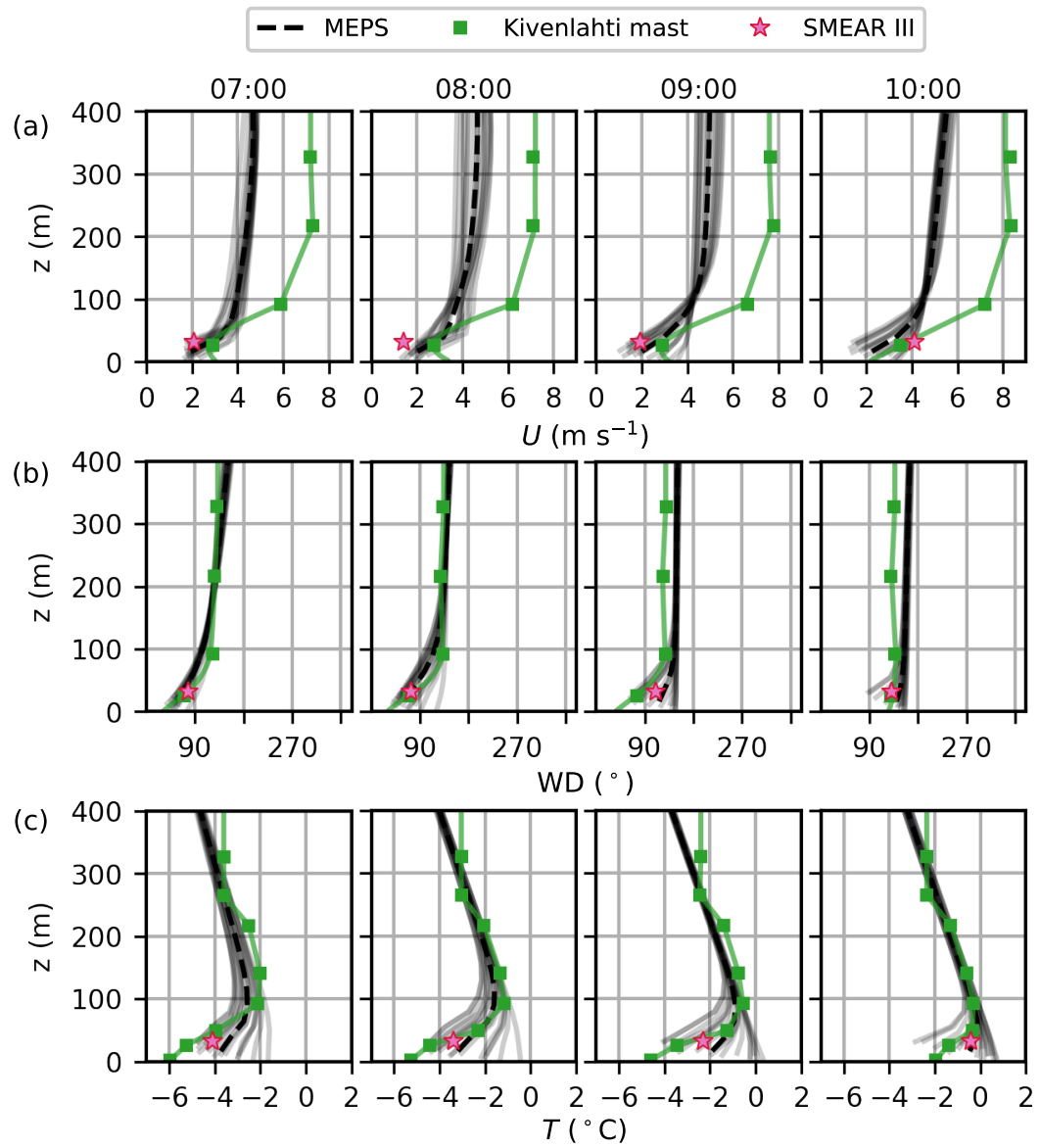

Figure S10. Horizontal a) wind speed $U\left(\mathrm{~m} \mathrm{~s}^{-1}\right)$, b) wind direction WD $\left({ }^{\circ}\right)$ and c) air temperature $T\left({ }^{\circ} \mathrm{C}\right)$ on 12 Dec at local time (UTC+3). See Fig. S5 caption for details.

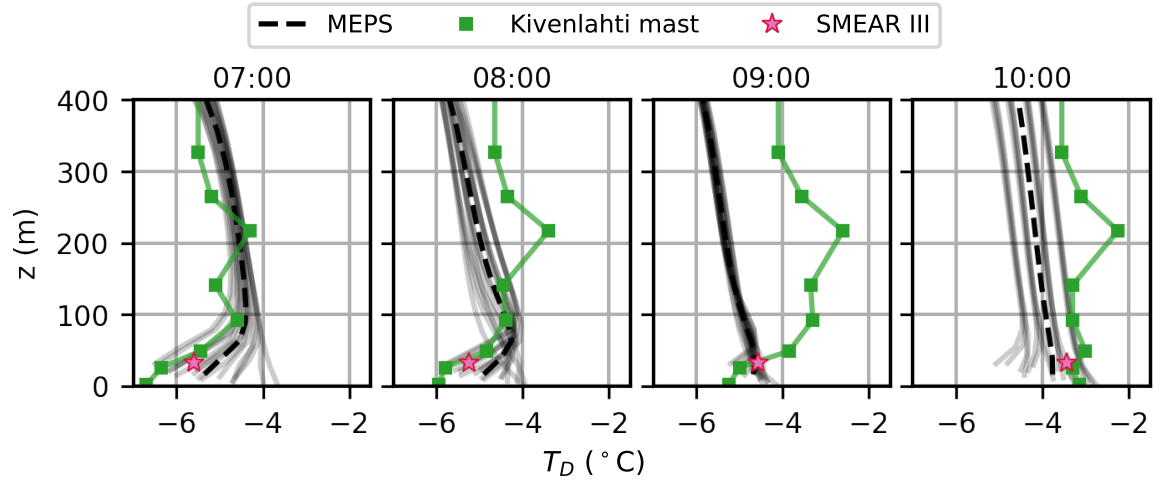

Figure S11. Dew-point temperature $T_{D}\left({ }^{\circ} \mathrm{C}\right)$ on $12 \mathrm{Dec}$ at local time (UTC+2). See Fig. S5 for details. 


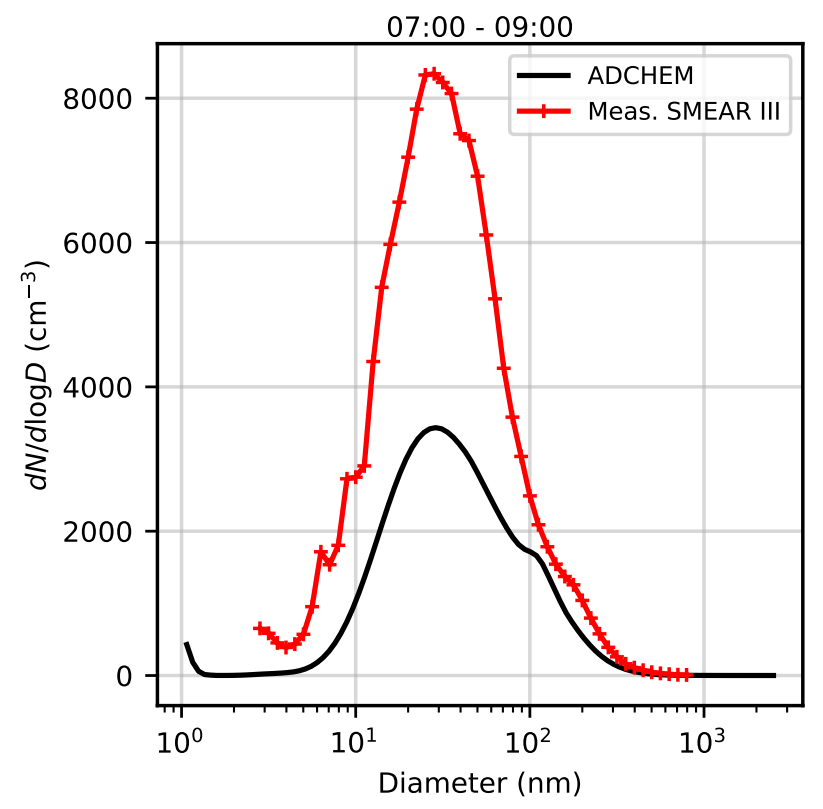

Figure S12. Mean modelled (black solid line) and measured aerosol size distribution at the SMEAR station (red line with crosses) on 12 Dec 2017 between 07:00-09:00 local time. Modelled values are from ADCHEM for a trajectory arriving in Helsinki $\left(60^{\circ} 12^{\prime} \mathrm{N}, 24^{\circ} 57^{\prime} \mathrm{E}\right)$.

\section{S5 Evaluation of the air quality modelling results}

\section{S5.2 Horizontal distribution of total aerosol particle number concentration}

Table S2. Significance of differences in the model absolute error (i.e., the difference between the modelled and observed values) compared to $\mathrm{M}_{\mathrm{MET}} \mathrm{M}_{\mathrm{PSD}}$ based on Student's t-test for the means of two independent samples.

\begin{tabular}{|c|c|c|c|}
\hline Simulation time & Simulation name & Significantly different? & $p$-value \\
\hline \multirow[t]{4}{*}{9 Jun, 07:16-08:14 } & $\mathrm{O}_{\mathrm{MET}} \mathrm{O}_{\mathrm{PSD}}$ & $-*$ & - \\
\hline & $\mathrm{M}_{\mathrm{MET}} \mathrm{O}_{\mathrm{PSD}}$ & $-{ }^{*}$ & - \\
\hline & $\mathrm{O}_{\mathrm{wD}, \text { mast }} \mathrm{O}_{\mathrm{PSD}}$ & $-*$ & - \\
\hline & $\mathrm{O}_{\text {WD,SMEAR }} \mathrm{O}_{\mathrm{PSD}}$ & No & 0.039 \\
\hline \multirow[t]{4}{*}{9 Jun, 08:23-09:15 } & $\mathrm{O}_{\mathrm{MET}} \mathrm{O}_{\mathrm{PSD}}$ & Yes & 0.80 \\
\hline & $\mathrm{M}_{\mathrm{MET}} \mathrm{O}_{\mathrm{PSD}}$ & Yes & 0.92 \\
\hline & $\mathrm{O}_{\mathrm{WD}, \text { mast }} \mathrm{O}_{\mathrm{PSD}}$ & Yes & 0.39 \\
\hline & $\mathrm{O}_{\text {WD,SMEAR }} \mathrm{O}_{\mathrm{PSD}}$ & $-^{*}$ & - \\
\hline 9 Jun, 20:26-21:14 & $\mathrm{O}_{\mathrm{MET}} \mathrm{O}_{\mathrm{PSD}}$ & Yes & 0.34 \\
\hline 12 Dec, 07:20-08:08 & $\mathrm{O}_{\text {MET }} \mathrm{O}_{\text {PSD }}$ & No & $2 \times 10^{-11}$ \\
\hline 12 Dec, 08:14-09:14 & $\mathrm{O}_{\text {MET }} \mathrm{O}_{\mathrm{PSD}}$ & $-^{*}$ & - \\
\hline
\end{tabular}

*: Variances are not homogeneous according to a Levene's test and a Student's t-test cannot be performed 
Table S3. Model performance for the aerosol size distribution for $\mathrm{M}_{\mathrm{MET}} \mathrm{M}_{\mathrm{PSD}}$ on 9 Jun morning. Performance measures: fractional bias (FB), normalised mean squared error (NMSE), factor of two (FAC2), normalised mean bias factor (NMBF) and normalised mean absolute error factor (NMAEF). Colours indicate whether the value fits (blue) or not (red) within the criteria in Table 6.

\begin{tabular}{|c|c|c|c|c|c|c|c|}
\hline Time & Measure & Instrument & Background & Side street & Main street & Opposite supersite & Supersite \\
\hline \multirow[t]{10}{*}{ 07:20-08:08 } & \multirow[t]{2}{*}{ FB } & EEPS & 0.04 & -0.85 & 0.23 & - & 0.36 \\
\hline & & ELPI & -0.26 & -1.32 & -0.05 & - & 0.20 \\
\hline & \multirow[t]{2}{*}{ NMSE } & EEPS & 3.12 & 3.30 & 1.61 & - & 1.06 \\
\hline & & ELPI & 1.49 & 6.76 & 1.16 & - & 0.72 \\
\hline & \multirow[t]{2}{*}{ FAC2 } & EEPS & 0.50 & 0.12 & 0.50 & - & 0.50 \\
\hline & & ELPI & 0.38 & 0.00 & 0.75 & - & 0.62 \\
\hline & \multirow[t]{2}{*}{ NMBF } & EEPS & 0.89 & -2.45 & 0.40 & - & 0.27 \\
\hline & & ELPI & 0.06 & -4.58 & 0.08 & - & 0.14 \\
\hline & \multirow[t]{2}{*}{ NMAEF } & EEPS & 1.24 & 2.63 & 0.88 & - & 0.78 \\
\hline & & ELPI & 0.86 & 4.58 & 0.62 & - & 0.57 \\
\hline \multirow[t]{10}{*}{ 08:14-09:14 } & \multirow[t]{2}{*}{ FB } & EEPS & - & -0.75 & 0.44 & 0.15 & 0.78 \\
\hline & & ELPI & - & -1.21 & 0.25 & 0.00 & 0.47 \\
\hline & \multirow[t]{2}{*}{ NMSE } & EEPS & - & 2.44 & 11.09 & 4.91 & 15.64 \\
\hline & & ELPI & - & 4.23 & 7.05 & 3.82 & 9.16 \\
\hline & \multirow[t]{2}{*}{ FAC2 } & EEPS & - & 0.12 & 0.25 & 0.50 & 0.38 \\
\hline & & ELPI & - & 0.12 & 0.50 & 0.62 & 0.62 \\
\hline & \multirow[t]{2}{*}{ NMBF } & EEPS & - & -1.51 & 2.34 & 0.84 & 4.09 \\
\hline & & ELPI & - & -2.99 & 1.33 & 0.42 & 2.33 \\
\hline & \multirow{2}{*}{ NMAEF } & EEPS & - & 2.04 & 2.76 & 1.34 & 4.15 \\
\hline & & ELPI & - & 2.99 & 1.91 & 1.24 & 2.60 \\
\hline
\end{tabular}


Table S4. Model performance for the aerosol size distribution for $\mathrm{O}_{\mathrm{MET}} \mathrm{O}_{\mathrm{PSD}}$ on 9 Jun morning. See Table $\mathrm{S} 3$ for further description.

\begin{tabular}{|c|c|c|c|c|c|c|c|}
\hline Time & Measure & Instrument & Background & Side street & Main street & Opposite supersite & Supersite \\
\hline \multirow[t]{10}{*}{ 07:20-08:08 } & \multirow[t]{2}{*}{ FB } & EEPS & 0.07 & -0.64 & 0.46 & - & 0.21 \\
\hline & & ELPI & -0.42 & -1.12 & 0.23 & - & 0.11 \\
\hline & \multirow[t]{2}{*}{ NMSE } & EEPS & 0.19 & 2.25 & 10.32 & - & 5.44 \\
\hline & & ELPI & 0.86 & 3.44 & 7.74 & - & 4.66 \\
\hline & \multirow[t]{2}{*}{ FAC2 } & EEPS & 0.62 & 0.00 & 0.12 & - & 0.38 \\
\hline & & ELPI & 0.50 & 0.25 & 0.25 & - & 0.38 \\
\hline & \multirow[t]{2}{*}{ NMBF } & EEPS & 0.07 & -1.11 & 2.11 & - & 0.64 \\
\hline & & ELPI & -0.68 & -2.38 & 1.42 & - & 0.50 \\
\hline & \multirow[t]{2}{*}{ NMAEF } & EEPS & 0.39 & 1.80 & 2.69 & - & 1.64 \\
\hline & & ELPI & 0.81 & 2.38 & 2.13 & - & 1.52 \\
\hline \multirow[t]{10}{*}{ 08:14-09:14 } & \multirow[t]{2}{*}{ FB } & EEPS & - & -0.68 & 0.45 & 0.57 & 0.13 \\
\hline & & ELPI & - & -1.14 & 0.17 & 0.38 & -0.27 \\
\hline & \multirow[t]{2}{*}{ NMSE } & EEPS & - & 2.33 & 11.53 & 9.65 & 3.09 \\
\hline & & ELPI & - & 3.86 & 7.39 & 7.09 & 1.68 \\
\hline & \multirow{2}{*}{ FAC2 } & EEPS & - & 0.12 & 0.25 & 0.12 & 0.50 \\
\hline & & ELPI & - & 0.25 & 0.38 & 0.38 & 0.62 \\
\hline & \multirow{2}{*}{ NMBF } & EEPS & - & -1.33 & 2.42 & 2.50 & 0.65 \\
\hline & & ELPI & - & -2.69 & 1.38 & 1.69 & 0.08 \\
\hline & \multirow[t]{2}{*}{ NMAEF } & EEPS & - & 1.92 & 2.85 & 2.86 & 1.05 \\
\hline & & ELPI & - & 2.69 & 2.02 & 2.18 & 0.77 \\
\hline
\end{tabular}


Table S5. Model performance for the aerosol size distribution for $\mathrm{M}_{\mathrm{MET}} \mathrm{O}_{\mathrm{PSD}}$ on 9 Jun morning. See Table S3 for further description.

\begin{tabular}{|c|c|c|c|c|c|c|c|}
\hline Time & Measure & Instrument & Background & Side street & Main street & Opposite supersite & Supersite \\
\hline \multirow[t]{10}{*}{ 07:20-08:08 } & \multirow[t]{2}{*}{ FB } & EEPS & 0.27 & -0.75 & 0.27 & - & 0.40 \\
\hline & & ELPI & -0.12 & -1.24 & -0.10 & - & 0.16 \\
\hline & \multirow[t]{2}{*}{ NMSE } & EEPS & 3.58 & 2.90 & 1.52 & - & 1.00 \\
\hline & & ELPI & 1.62 & 6.06 & 1.10 & - & 0.68 \\
\hline & \multirow[t]{2}{*}{ FAC2 } & EEPS & 0.50 & 0.12 & 0.50 & - & 0.38 \\
\hline & & ELPI & 0.38 & 0.12 & 0.62 & - & 0.62 \\
\hline & \multirow[t]{2}{*}{ NMBF } & EEPS & 1.14 & -2.16 & 0.40 & - & 0.28 \\
\hline & & ELPI & 0.20 & -4.11 & 0.08 & - & 0.15 \\
\hline & \multirow[t]{2}{*}{ NMAEF } & EEPS & 1.41 & 2.35 & 0.86 & - & 0.78 \\
\hline & & ELPI & 0.88 & 4.11 & 0.61 & - & 0.57 \\
\hline \multirow[t]{10}{*}{ 08:14-09:14 } & \multirow[t]{2}{*}{ FB } & EEPS & - & -0.67 & 0.46 & 0.20 & 0.81 \\
\hline & & ELPI & - & -1.14 & 0.17 & -0.04 & 0.41 \\
\hline & \multirow{2}{*}{ NMSE } & EEPS & - & 2.27 & 11.00 & 4.83 & 15.45 \\
\hline & & ELPI & - & 3.94 & 7.00 & 3.77 & 9.05 \\
\hline & \multirow{2}{*}{ FAC2 } & EEPS & - & 0.12 & 0.25 & 0.38 & 0.12 \\
\hline & & ELPI & - & 0.25 & 0.38 & 0.50 & 0.38 \\
\hline & \multirow{2}{*}{ NMBF } & EEPS & - & -1.37 & 2.35 & 0.87 & 4.15 \\
\hline & & ELPI & - & -2.77 & 1.34 & 0.44 & 2.37 \\
\hline & \multirow[t]{2}{*}{ NMAEF } & EEPS & - & 1.91 & 2.78 & 1.35 & 4.21 \\
\hline & & ELPI & - & 2.77 & 1.93 & 1.26 & 2.64 \\
\hline
\end{tabular}


Table S6. Model performance for the aerosol size distribution for $\mathrm{O}_{\mathrm{WD} \text {,mast }} \mathrm{O}_{\mathrm{PSD}}$ on 9 Jun morning. See Table S3 for further description.

\begin{tabular}{|c|c|c|c|c|c|c|c|}
\hline Time & Measure & Instrument & Background & Side street & Main street & Opposite supersite & Supersite \\
\hline \multirow[t]{10}{*}{ 07:20-08:08 } & \multirow[t]{2}{*}{ FB } & EEPS & 0.20 & -0.73 & 0.44 & - & 0.27 \\
\hline & & ELPI & -0.22 & -1.21 & 0.19 & - & 0.18 \\
\hline & \multirow[t]{2}{*}{ NMSE } & EEPS & 1.56 & 2.62 & 8.49 & - & 6.31 \\
\hline & & ELPI & 0.89 & 5.23 & 6.32 & - & 5.39 \\
\hline & \multirow[t]{2}{*}{ FAC2 } & EEPS & 0.50 & 0.12 & 0.25 & - & 0.38 \\
\hline & & ELPI & 0.50 & 0.25 & 0.38 & - & 0.38 \\
\hline & \multirow[t]{2}{*}{ NMBF } & EEPS & 0.70 & -1.86 & 1.78 & - & 0.88 \\
\hline & & ELPI & -0.05 & -3.59 & 1.17 & - & 0.72 \\
\hline & \multirow[t]{2}{*}{ NMAEF } & EEPS & 0.96 & 2.17 & 2.35 & - & 1.82 \\
\hline & & ELPI & 0.71 & 3.59 & 1.82 & - & 1.68 \\
\hline \multirow[t]{10}{*}{ 08:14-09:14 } & \multirow[t]{2}{*}{ FB } & EEPS & - & -0.62 & 0.51 & 0.58 & 0.18 \\
\hline & & ELPI & - & -1.07 & 0.21 & 0.33 & -0.34 \\
\hline & \multirow[t]{2}{*}{ NMSE } & EEPS & - & 2.31 & 10.85 & 6.77 & 1.41 \\
\hline & & ELPI & - & 2.89 & 6.88 & 4.90 & 0.87 \\
\hline & \multirow[t]{2}{*}{ FAC2 } & EEPS & - & 0.00 & 0.12 & 0.25 & 0.62 \\
\hline & & ELPI & - & 0.25 & 0.38 & 0.50 & 0.62 \\
\hline & \multirow[t]{2}{*}{ NMBF } & EEPS & - & -0.85 & 2.43 & 1.94 & 0.31 \\
\hline & & ELPI & - & -1.93 & 1.39 & 1.26 & -0.19 \\
\hline & \multirow[t]{2}{*}{ NMAEF } & EEPS & - & 1.59 & 2.83 & 2.25 & 0.68 \\
\hline & & ELPI & - & 1.93 & 1.92 & 1.71 & 0.65 \\
\hline
\end{tabular}


Table S7. Model performance for the aerosol size distribution for $\mathrm{O}_{W D, S M E A R} \mathrm{O}_{\mathrm{PSD}}$ on 9 Jun morning. See Table S3 for further description.

\begin{tabular}{|c|c|c|c|c|c|c|c|}
\hline Time & Measure & Instrument & Background & Side street & Main street & Opposite supersite & Supersite \\
\hline \multirow[t]{10}{*}{ 07:20-08:08 } & \multirow[t]{2}{*}{ FB } & EEPS & 0.21 & -0.68 & 0.40 & - & 0.57 \\
\hline & & ELPI & -0.14 & -1.15 & 0.13 & - & 0.50 \\
\hline & \multirow[t]{2}{*}{ NMSE } & EEPS & 4.24 & 2.29 & 6.44 & - & 12.04 \\
\hline & & ELPI & 1.97 & 4.31 & 4.76 & - & 10.53 \\
\hline & \multirow[t]{2}{*}{ FAC2 } & EEPS & 0.50 & 0.12 & 0.38 & - & 0.12 \\
\hline & & ELPI & 0.38 & 0.25 & 0.38 & - & 0.38 \\
\hline & \multirow[t]{2}{*}{ NMBF } & EEPS & 1.27 & -1.48 & 1.40 & - & 2.35 \\
\hline & & ELPI & 0.26 & -2.98 & 0.87 & - & 2.08 \\
\hline & \multirow[t]{2}{*}{ NMAEF } & EEPS & 1.56 & 1.94 & 1.95 & - & 3.08 \\
\hline & & ELPI & 1.00 & 2.98 & 1.50 & - & 2.73 \\
\hline \multirow[t]{10}{*}{$08: 14-09: 14$} & \multirow[t]{2}{*}{ FB } & EEPS & - & -0.65 & 0.66 & 0.21 & 1.132 \\
\hline & & ELPI & - & -1.12 & 0.35 & -0.02 & 0.72 \\
\hline & \multirow[t]{2}{*}{ NMSE } & EEPS & - & 2.19 & 13.25 & 5.08 & 34.93 \\
\hline & & ELPI & - & 3.73 & 8.46 & 3.99 & 21.75 \\
\hline & \multirow[t]{2}{*}{ FAC2 } & EEPS & - & 0.12 & 0.25 & 0.38 & 0.25 \\
\hline & & ELPI & - & 0.25 & 0.25 & 0.50 & 0.38 \\
\hline & \multirow[t]{2}{*}{ NMBF } & EEPS & - & -1.28 & 3.15 & 0.89 & 8.49 \\
\hline & & ELPI & - & -2.62 & 1.89 & 0.45 & 5.21 \\
\hline & \multirow[t]{2}{*}{ NMAEF } & EEPS & - & 1.85 & 3.47 & 1.44 & 8.50 \\
\hline & & ELPI & - & 2.62 & 2.24 & 1.32 & 5.35 \\
\hline
\end{tabular}

Table S8. Model performance for the aerosol size distribution for $M_{M E T} M_{P S D}$ on 9 Jun evening. See Table S3 for further description.

\begin{tabular}{llllllll}
\hline Time & Measure & Instrument & Background & Side street & Main street & Opposite supersite & Supersite \\
\hline 20:26-21:14 & \multirow{2}{*}{ FB } & EEPS & -0.70 & -0.54 & 0.26 & -0.10 & 1.02 \\
& & ELPI & -0.58 & -0.75 & -0.15 & -0.16 & 1.20 \\
& \multirow{2}{*}{ NMSE } & EEPS & 0.70 & 1.24 & 0.54 & 0.46 & 7.61 \\
& \multirow{2}{*}{ FAC2 } & ELPI & 1.77 & 1.78 & 0.14 & 0.70 & 18.96 \\
& EEPS & 0.50 & 0.38 & 0.62 & 0.38 & 0.25 \\
& NMBF & ELPI & 0.38 & 0.38 & 0.88 & 0.38 & 0.12 \\
& EEPS & -0.60 & -1.00 & 0.28 & -0.17 & 3.63 \\
& ELPI & -0.96 & -1.38 & -0.05 & -0.11 & 8.89 \\
& \multirow{2}{*}{ NMAEF } & EEPS & 0.85 & 1.11 & 0.54 & 0.62 & 3.69 \\
& ELPI & 1.24 & 1.46 & 0.27 & 0.60 & 8.89 \\
\hline
\end{tabular}


Table S9. Model performance for the aerosol size distribution for $\mathrm{O}_{\mathrm{MET}} \mathrm{O}_{\mathrm{PSD}}$ on 9 Jun evening. See Table S3 for further description.

\begin{tabular}{llllllll}
\hline Time & Measure & Instrument & Background & Side street & Main street & Opposite supersite & Supersite \\
\hline 20:26-21:14 & \multirow{2}{*}{ FB } & EEPS & -0.15 & -0.37 & 0.34 & 0.51 & 1.03 \\
& & ELPI & -0.25 & -0.72 & -0.21 & 0.26 & 1.01 \\
& \multirow{2}{*}{ NMSE } & EEPS & 0.48 & 1.01 & 0.42 & 1.17 & 4.57 \\
& ELPI & 0.78 & 1.59 & 0.07 & 1.27 & 12.56 \\
& \multirow{2}{*}{ FAC2 } & EEPS & 0.50 & 0.50 & 0.75 & 0.50 & 0.25 \\
& ELPI & 0.75 & 0.50 & 0.88 & 0.62 & 0.25 \\
& \multirow{2}{*}{ NMBF } & EEPS & -0.01 & -0.97 & 0.27 & 0.73 & 6.68 \\
& ELPI & -0.24 & -1.35 & 0.08 & 0.82 & 2.68 \\
& NMAEF & EEPS & 0.45 & 1.01 & 0.45 & 0.92 & 6.72 \\
\hline
\end{tabular}

Table S10. Model performance for the aerosol size distribution for $\mathrm{M}_{\mathrm{MET}} \mathrm{M}_{\mathrm{PSD}}$ on $7 \mathrm{Dec}$ morning. See Table S3 for further description.

\begin{tabular}{|c|c|c|c|c|c|c|c|}
\hline Time & Measure & Instrument & Background & Side street & Main street & Opposite supersite & Supersite \\
\hline \multirow[t]{10}{*}{ 07:20-08:08 } & \multirow[t]{2}{*}{ FB } & EEPS & -0.02 & -0.65 & 0.27 & - & -0.34 \\
\hline & & ELPI & -0.02 & -0.83 & -0.02 & - & -0.54 \\
\hline & \multirow[t]{2}{*}{ NMSE } & EEPS & 0.64 & 2.71 & 3.08 & - & 1.70 \\
\hline & & ELPI & 1.56 & 3.38 & 4.58 & - & 1.41 \\
\hline & \multirow[t]{2}{*}{ FAC2 } & EEPS & 0.62 & 0.38 & 0.12 & - & 0.50 \\
\hline & & ELPI & 0.62 & 0.38 & 0.25 & - & 0.38 \\
\hline & \multirow[t]{2}{*}{$\mathrm{NMBF}$} & EEPS & 0.17 & -1.93 & 0.27 & - & -1.04 \\
\hline & & ELPI & 0.53 & -1.45 & 0.79 & - & -0.33 \\
\hline & \multirow[t]{2}{*}{ NMAEF } & EEPS & 0.58 & 1.95 & 1.23 & - & 1.28 \\
\hline & & ELPI & 0.82 & 1.91 & 1.51 & - & 0.93 \\
\hline \multirow[t]{10}{*}{$08: 14-09: 14$} & \multirow[t]{2}{*}{$\mathrm{FB}$} & EEPS & -0.82 & -0.64 & 0.03 & -0.63 & -0.28 \\
\hline & & ELPI & -1.05 & -0.87 & -0.23 & -1.02 & -0.47 \\
\hline & \multirow[t]{2}{*}{ NMSE } & EEPS & 1.93 & 2.70 & 5.21 & 8.61 & 3.13 \\
\hline & & ELPI & 2.49 & 3.96 & 7.78 & 8.42 & 3.37 \\
\hline & \multirow[t]{2}{*}{ FAC2 } & EEPS & 0.25 & 0.38 & 0.25 & 0.25 & 0.38 \\
\hline & & ELPI & 0.12 & 0.25 & 0.38 & 0.25 & 0.50 \\
\hline & \multirow[t]{2}{*}{ NMBF } & EEPS & -1.69 & -1.67 & 0.36 & -3.97 & -0.86 \\
\hline & & ELPI & -1.30 & -1.26 & 0.97 & -2.92 & -0.22 \\
\hline & \multirow[t]{2}{*}{ NMAEF } & EEPS & 1.70 & 1.68 & 1.51 & 3.98 & 1.64 \\
\hline & & ELPI & 1.82 & 2.01 & 1.98 & 3.11 & 1.25 \\
\hline
\end{tabular}


Table S11. Model performance for the aerosol size distribution for $\mathrm{O}_{\mathrm{MET}} \mathrm{O}_{\text {PSD }}$ on $7 \mathrm{Dec}$. See Table $\mathrm{S} 3$ for further description.

\begin{tabular}{|c|c|c|c|c|c|c|c|}
\hline Time & Measure & Instrument & Background & Side street & Main street & Opposite supersite & Supersite \\
\hline \multirow[t]{10}{*}{ 07:20-08:08 } & \multirow[t]{2}{*}{ FB } & EEPS & 0.67 & 0.05 & 0.31 & - & 0.33 \\
\hline & & ELPI & 0.46 & -0.23 & 0.04 & - & 0.12 \\
\hline & \multirow[t]{2}{*}{ NMSE } & EEPS & 4.30 & 0.85 & 5.90 & - & 3.89 \\
\hline & & ELPI & 6.84 & 1.62 & 8.84 & - & 7.04 \\
\hline & \multirow[t]{2}{*}{ FAC2 } & EEPS & 0.38 & 0.50 & 0.00 & - & 0.25 \\
\hline & & ELPI & 0.50 & 0.62 & 0.25 & - & 0.50 \\
\hline & \multirow[t]{2}{*}{ NMBF } & EEPS & 2.05 & 0.11 & 0.74 & - & 0.76 \\
\hline & & ELPI & 2.98 & 0.33 & 1.45 & - & 1.73 \\
\hline & \multirow[t]{2}{*}{ NMAEF } & EEPS & 2.12 & 0.60 & 1.81 & - & 1.40 \\
\hline & & ELPI & 3.03 & 0.81 & 2.32 & - & 2.05 \\
\hline \multirow[t]{10}{*}{ 08:14-09:14 } & \multirow[t]{2}{*}{ FB } & EEPS & 0.08 & 0.02 & 0.38 & 0.16 & 0.16 \\
\hline & & ELPI & -0.42 & -0.38 & 0.15 & -0.12 & -0.09 \\
\hline & \multirow[t]{2}{*}{ NMSE } & EEPS & 0.42 & 0.15 & 10.69 & 4.06 & 1.38 \\
\hline & & ELPI & 0.38 & 0.48 & 16.27 & 6.92 & 1.84 \\
\hline & \multirow[t]{2}{*}{ FAC2 } & EEPS & 0.75 & 0.62 & 0.25 & 0.12 & 0.38 \\
\hline & & ELPI & 0.62 & 0.50 & 0.38 & 0.12 & 0.50 \\
\hline & \multirow[t]{2}{*}{ NMBF } & EEPS & 0.04 & -0.17 & 1.75 & 0.23 & -0.06 \\
\hline & & ELPI & 0.20 & -0.01 & 2.97 & 0.56 & 0.44 \\
\hline & \multirow[t]{2}{*}{ NMAEF } & EEPS & 0.38 & 0.25 & 2.67 & 1.41 & 0.74 \\
\hline & & ELPI & 0.45 & 0.41 & 3.66 & 1.92 & 0.88 \\
\hline
\end{tabular}




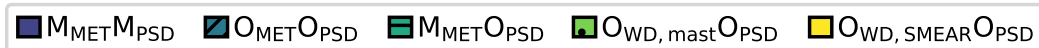
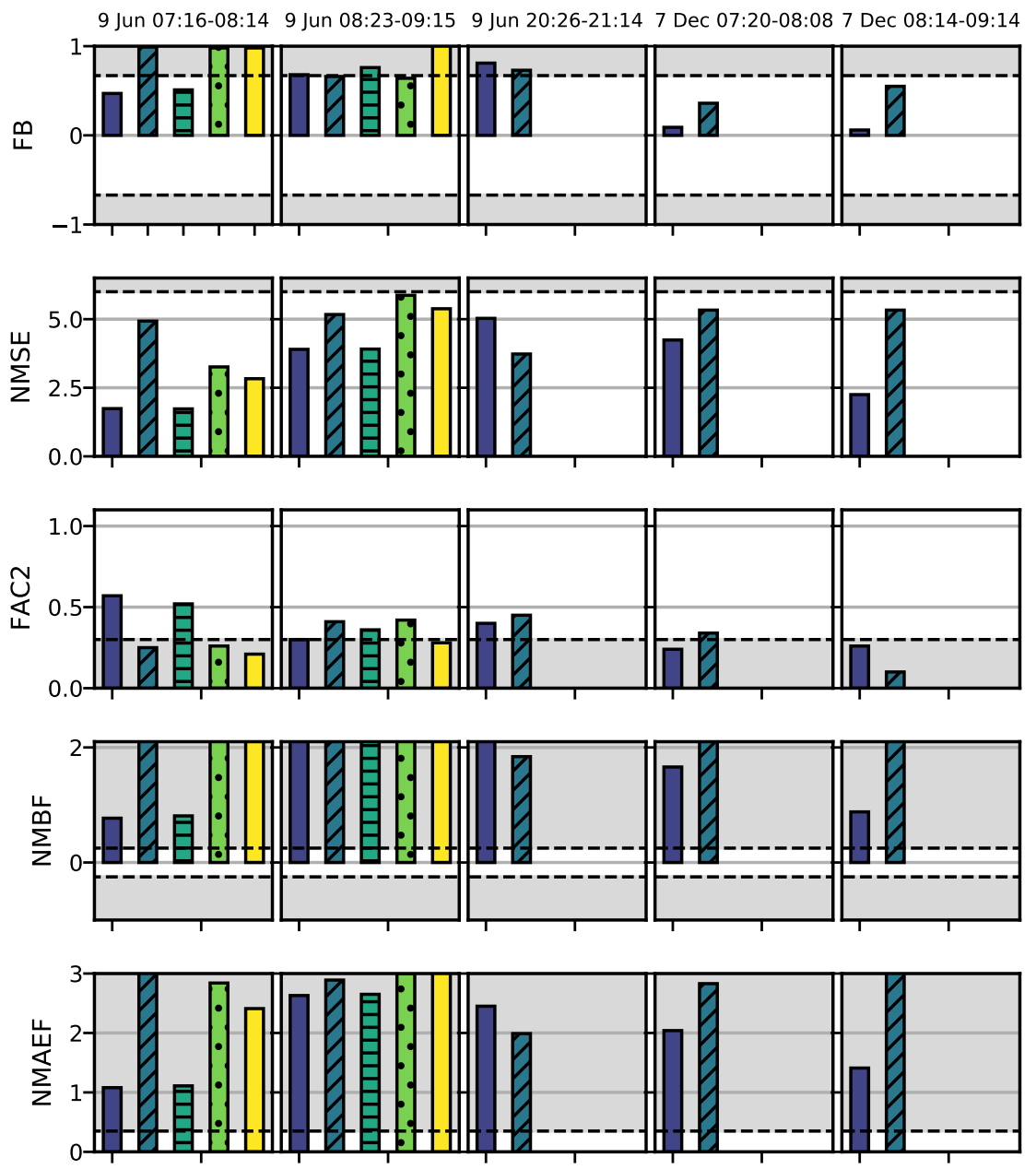

Figure S13. Model performance for the horizontal distribution of BC. See Table S3 for further description. 
Table S12. Performance of the modelled aerosol chemical composition at the supersite on 9 Jun evening between 20:26-21:14. See Table S3 for further description.

\begin{tabular}{lllllll}
\hline Simulation name & Variable & FB & NMSE & FAC2 & NMBF & NMAEF \\
\hline $\mathrm{M}_{\text {MET }} \mathrm{M}_{\text {PSD }}$ & $\mathrm{SO}_{4}^{-}$ & 0.44 & 0.21 & 1.00 & 0.57 & 0.57 \\
& $\mathrm{OC}$ & -0.29 & 0.11 & 1.00 & -0.34 & 0.34 \\
& $\mathrm{NO}_{3}^{-}$ & 1.09 & 1.69 & 0.00 & 2.39 & 2.39 \\
& $\mathrm{NH}_{4}^{+}$ & 1.20 & 2.49 & 0.00 & 3.12 & 3.12 \\
& $\mathrm{BC}$ & 1.17 & 2.18 & 0.00 & 2.85 & 2.85 \\
& $\mathrm{PM}_{2.5}$ & 0.38 & 0.17 & 1.00 & 0.47 & 0.47 \\
$\mathrm{O}_{\text {MET }} \mathrm{O}_{\text {PSD }}$ & 0.81 & 0.78 & 0.00 & 1.36 & 1.36 \\
& $\mathrm{OC}_{4}^{-}$ & 0.13 & 0.02 & 1.00 & 0.13 & 0.13 \\
& $\mathrm{NO}_{3}^{-}$ & 1.27 & 2.73 & 0.00 & 3.51 & 3.51 \\
& $\mathrm{NH}_{4}^{+}$ & 1.58 & 6.79 & 0.00 & 7.55 & 7.55 \\
& $\mathrm{BC}$ & 1.48 & 4.89 & 0.00 & 5.70 & 5.70 \\
& $\mathrm{PM}_{2.5}$ & 0.83 & 0.85 & 0.00 & 1.42 & 1.42 \\
\hline
\end{tabular}

Table S13. Performance of the modelled aerosol chemical composition at the supersite on 12 Dec morning between 07:20-09:14. See Table S3 for further description.

\begin{tabular}{lllllll}
\hline Simulation name & Variable & FB & NMSE & FAC2 & NMBF & NMAEF \\
\hline $\mathrm{M}_{\text {MET MPSD }}$ & $\mathrm{SO}_{4}^{-}$ & 1.21 & 2.59 & 0.00 & 3.18 & 3.18 \\
& $\mathrm{OC}$ & -0.41 & 0.20 & 1.00 & -0.52 & 0.52 \\
& $\mathrm{NO}_{3}^{-}$ & 1.13 & 1.89 & 0.00 & 2.60 & 2.60 \\
& $\mathrm{NH}_{4}^{+}$ & 1.40 & 5.75 & 0.00 & 5.48 & 5.48 \\
& $\mathrm{BC}$ & 0.87 & 1.37 & 0.33 & 1.71 & 1.71 \\
$\mathrm{PM}_{2.5}$ & 0.54 & 0.41 & 0.67 & 0.78 & 0.78 \\
$\mathrm{O}_{\text {MET }} \mathrm{O}_{\mathrm{PSD}}$ & $\mathrm{SO}_{4}^{-}$ & 1.84 & 21.50 & 0.00 & 22.21 & 22.21 \\
& $\mathrm{OC}$ & 0.72 & 0.58 & 0.50 & 1.06 & 1.06 \\
& $\mathrm{NO}_{3}^{-}$ & 0.87 & 1.03 & 0.17 & 1.57 & 1.57 \\
& $\mathrm{NH}_{4}^{+}$ & 1.63 & 7.46 & 0.00 & 7.62 & 7.62 \\
& $\mathrm{BC}$ & 0.11 & 0.22 & 0.67 & 0.20 & 0.46 \\
& $\mathrm{PM}_{2.5}$ & 0.88 & 1.04 & 0.50 & 1.52 & 1.52 \\
\hline
\end{tabular}




\section{S6 Sensitivity analysis}
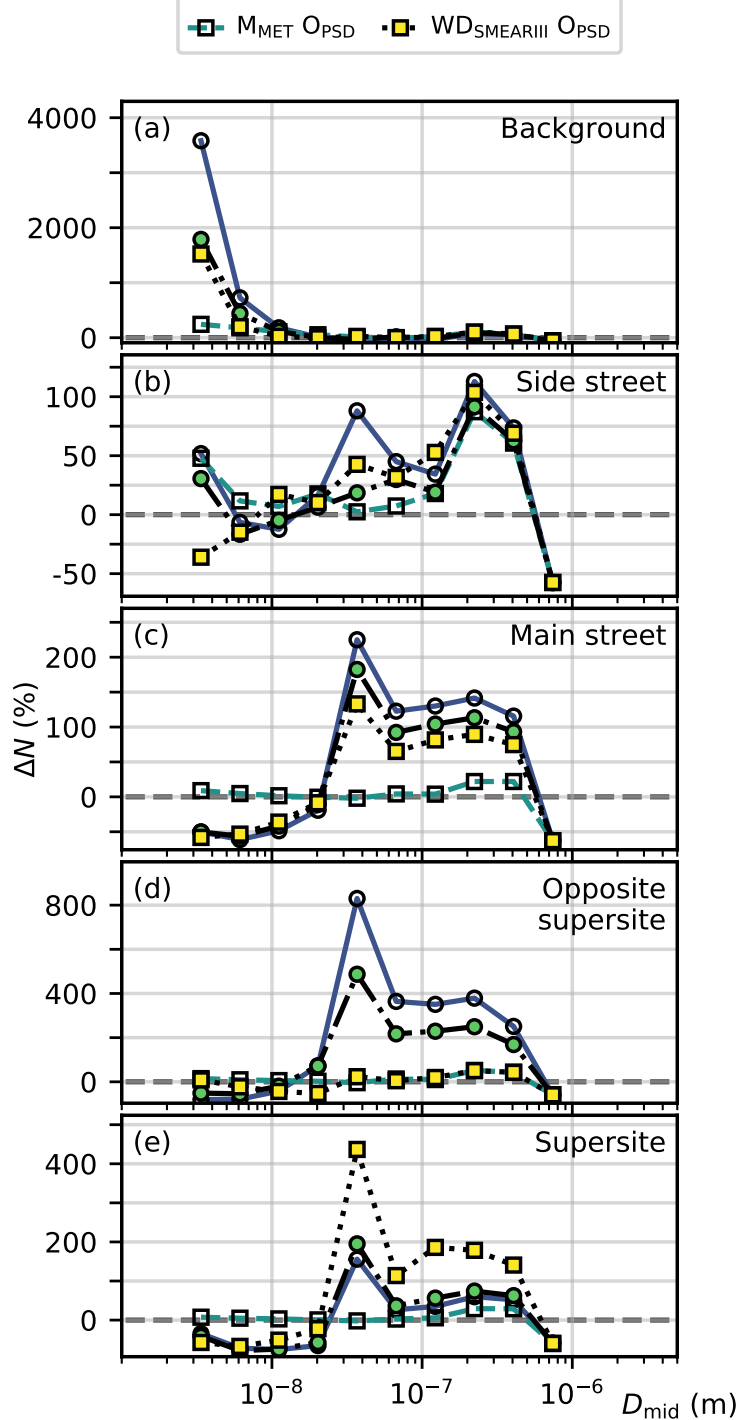

Figure S14. Relative difference in the aerosol number concentration $\Delta N(\%)$ compared to $\mathrm{M}_{\mathrm{MET}} \mathrm{M}_{\mathrm{PSD}}$ to as a function of aerosol particle diameter $D_{\text {mid }}(\mathrm{nm})$ at $z=1.5 \mathrm{~m}$ on 9 Jun at 07:16-08:14. The figure shows the difference for $\mathrm{O}_{\mathrm{MET}} \mathrm{O}_{\mathrm{PSD}}$ (solid line with empty circles), $\mathrm{M}_{\text {MET }} \mathrm{O}_{\text {PSD }}$ (dashed line with empty squares), $\mathrm{O}_{\mathrm{WD} \text {,mast }} \mathrm{O}_{\mathrm{PSD}}$ (dash-dot line with filled squares) and $\mathrm{O}_{\mathrm{WD}, \mathrm{SMEAR}} \mathrm{O}_{\mathrm{PSD}}$ (dotted line with filled squares). 


\section{References}

Granier, C., Darras, S., Denier van der Gon, H., Doubalova, J., Elguindi, N., Galle, B., Gauss, M., Guevara, M., Jalkanen, J.-P., Kuenen, J., Liousse, C., Quack, B., Simpson, D., and Sindelarova, K.: The Copernicus Atmosphere Monitoring Service global and regional emissions (April 2019 version), https://doi.org/10.24380/d0bn-kx16, 2019.

5 Guenther, A., Karl, T., Harley, P., Wiedinmyer, C., Palmer, P. I., and Geron, C.: Estimates of global terrestrial isoprene emissions using MEGAN (Model of Emissions of Gases and Aerosols from Nature), Atmos. Chem. Phys., 6, 3181-3210, https://doi.org/10.5194/acp-63181-2006, 2006.

Karvosenoja, N.: Emission scenario model for regional air pollution, Tech. rep., Finnish Environment Institute, Helsinki, Finland, 2008.

Kristensson, A., Johansson, C., Westerholm, R., Swietlicki, E., Gidhagen, L., Wideqvist, U., and Vesely, V.: Real-world traffic emission factors of gases and particles measured in a road tunnel in Stockholm, Sweden, Atmos. Environ., 38, 657-673, https://doi.org/10.1016/j.atmosenv.2003.10.030, 2004.

Lana, A., Bell, T. G., Simó, R., Vallina, S. M., Ballabrera-Poy, J., Kettle, A. J., Dachs, J., Bopp, L., Saltzman, E. S., Stefels, J., Johnson, J. E., and Liss, P. S.: An updated climatology of surface dimethlysulfide concentrations and emission fluxes in the global ocean, Global Biogeochem. Cy., 25, https://doi.org/10.1029/2010GB003850, 2011.

15 Mårtensson, E. M., Nilsson, E. D., de Leeuw, G., Cohen, L. H., and Hansson, H.-C.: Laboratory simulations and parameterization of the primary marine aerosol production, J. Geophys. Res.-Atmos., 108, https://doi.org/10.1029/2002JD002263, 2003.

Paasonen, P., Kupiainen, K., Klimont, Z., Visschedijk, A., Denier van der Gon, H. A. C., and Amann, M.: Continental anthropogenic primary particle number emissions, Atmos. Chem. Phys., 16, 6823-6840, https://doi.org/10.5194/acp-16-6823-2016, 2016.

Ripamonti, G., Järvi, L., Mølgaard, B., Hussein, T., Nordbo, A., and Hämeri, K.: The effect of local sources on aerosol particle number size distribution, concentrations and fluxes in Helsinki, Finland, Tellus B Chem. Phys. Meteorol., 65, 19786, https://doi.org/10.3402/tellusb.v65i0.19786, 2013.

Roldin, P., Ehn, M., Kurtén, T., Olenius, T., Rissanen, M. P., Sarnela, N., Elm, J., Rantala, P., Hao, L., Hyttinen, N., et al.: The role of highly oxygenated organic molecules in the Boreal aerosol-cloud-climate system, Nat. Commun., 10, 1-15, 2019.

Schaap, M., Timmermans, R. M., Roemer, M., Boersen, G., Builtjes, P., Sauter, F., Velders, G., and Beck, J.: The LOTOS-EUROS model: description, validation and latest developments, Int. J. Environ. Pollut., 32, 270-290, 2008.

Simpson, D., Andersson, C., Christensen, J. H., Engardt, M., Geels, C., Nyiri, A., Posch, M., Soares, J., Sofiev, M., Wind, P., and Langner, J.: Impacts of climate and emission changes on nitrogen deposition in Europe: a multi-model study, Atmos. Chem. Phys., 14, 6995-7017, https://doi.org/10.5194/acp-14-6995-2014, 2014.

Wang, F., Ketzel, M., Ellermann, T., Wåhlin, P., Jensen, S. S., Fang, D., and Massling, A.: Particle number, particle mass and NO ${ }_{x}$ emission factors at a highway and an urban street in Copenhagen, Atmos. Chemi. Phys., 10, 2745-2764, https://doi.org/10.5194/acp-10-2745-2010, 2010. 